\title{
The edge waves on a Kirchhoff plate bilaterally supported by a two-parameter elastic foundation
}

\author{
J. Kaplunov*, A. Nobili ${ }^{\dagger}$
}

\begin{abstract}
In this paper, the bending waves propagating along the edge of a semi-infinite Kirchhoff plate resting on a two-parameter Pasternak elastic foundation are studied. Two geometries of the foundation are considered: either it is infinite or it is semi-infinite with the edges of the plate and of the foundation coinciding. Dispersion relations along with phase and group velocity expressions are obtained. It is shown that the semi-infinite foundation setup exhibits a cut-off frequency which is the same as for a Winkler foundation. The phase velocity possesses a
\end{abstract}

*School of Computing and Mathematics, Keele University, Keele, UK

†Dipartimento di Ingegneria Enzo Ferrari, Università di Modena e Reggio Emilia, Modena, Italy Corresponding author: Andrea Nobili, Dipartimento di Ingegneria Enzo Ferrari, Università di Modena e Reggio Emilia, via Vignolese 905, 41122, Modena, Italy, Email: andrea.nobili@unimore.it 
minimum which corresponds to the critical velocity of a moving load. The infinite foundation exhibits a cut-off frequency which depends on its relative stiffness and occurs at a nonzero wavenumber, which fact is hardly observed in elastodynamics. As a result, the associated phase velocity minimum is admissible only up to a limiting value of the stiffness. In the case of a foundation with small stiffness, asymptotic expansions are derived and beam-like one-dimensional equivalent models are deduced accordingly. It is demonstrated that for the infinite foundation the related non-classical beam-like model comprises a pseudo-differential operator.

Keywords: Edge wave; Kirchhoff plate; Pasternak foundation; Moving load; Dispersion

\section{Introduction}

Elastic waves localized at the edge of thin-walled structures, commonly named edge waves, may be regarded as a generalization of the classical Rayleigh waves. Edge waves belong to the realm of Surface Acoustic Waves (SAW) which has stimulated a great deal of interest in light of its applications in the context of signal processing devices, nondestructive evaluation (NE), seismology (Biryukov et al., 1995). White and Voltmer (1965) have been successfully employing surface waves through direct piezoelectric coupling in several microelectronic applications, ranging from sensing and actuation to telecommunications and biosensors (White, 1970). SAW appear to play a pivotal 
role in a wide array of natural phenomena such as the excitation the outer hair cells of the cochlea (Bell, 2006) or in beach cusps pattern formation (Sallenger Jr, 1979).

Edge waves were first shown to exist theoretically by Konenkov (1960) and several time independently rediscovered afterward (Norris et al., 2000). It is remarkable that a similar problem dealing with localized stability of a semiinfinite elastic plate was considered even earlier by Ishlinskii (1954). Elastic waves at the edge of plates and shells were intensively studied under fairly broad assumptions taking into account the effects of pre-stress, anisotropy, curvature and transverse inhomogeneity, e.g. see an overview by Lawrie and Kaplunov (2012) and references therein. However, until the recent note by Kaplunov et al. (2014), elastically supported structures were not tackled. The edge bending wave on a semi-infinite Kirchhoff plate resting on a Winkler foundation, considered in Kaplunov et al. (2014), exhibits a cut-off frequency along with a minimum for the phase velocity which corresponds to the critical speed of a moving load, in a similar manner as for the classical problem for a beam supported by an elastic foundation, e.g. see Timoshenko (1926).

In this paper we extend the considerations of Kaplunov et al. (2014) to a more general two parameter elastic foundation model, known as the Pasternak foundation (Selvadurai, 1979). The Pasternak foundation is often termed "weakly non-local" because it takes into account the effect of an infinitesimally small neighborhood of the material when expressing the foundation response at a point (Nobili, 2012a). This in contrast to the purely local foundation models, such as Winkler's, on the one hand, and to the fully non- 
local models, such as Wieghardt (1922)'s, on the other hand. In fact, the latter expresses the foundation response through an integral of the overall soil behavior. Introducing a non-local soil response has a threefold purpose. First of all, it is more realistic and better approximates the sophisticated behavior of a substrate. Besides, it addresses the question whether the reduction to a one dimensional problem is in fact due to the very special nature of the foundation. In addition, the effect of plate boundary conditions (BCs) other than those for a free edge is brought into evidence.

Here, two layouts and accordingly two sets of BCs are analyzed. A first set of BCs corresponds to a semi-infinite Pasternak foundation whose edge coincides with the edge of the plate. A second set of BCs is concerned with an infinite foundation and is analogous in a sense to the sophisticated threedimensional problem for a plate lying on an acoustic half-space. The two sets of BCs are determined via variational arguments in Nobili (2012b). The dispersion relations are derived and investigated for both aforementioned cases. The main focus is on the qualitative and quantitative analysis of the cut-off frequencies and of the minima of the phase velocity.

Asymptotic expansions are obtained in terms of a dimensionless small parameter $\eta$ expressing a ratio of foundation and plate stiffnesses. The practically important situation in which $\eta$ tends to zero represents, for instance, a small deviation from a local (Winkler) foundation. In the case of a semiinfinite soil, such expansions are shown to correspond to a one-dimensional equivalent formulation for a beam resting on a Pasternak elastic foundation with certain effective properties, see Kaplunov and Prikazchikov (2013) 
for more detail. Besides, a cut-off occurs at the same frequency as for the Winkler foundation. The infinite foundation scenario appears to be more involved. Indeed, the cut-off frequency of the edge wave depends on $\eta$ and it corresponds to a non-zero wavenumber. As a result, the phase velocity minimum is admissible only up to a limiting value of $\eta$. More surprisingly, already at the first order deviation from the Winkler foundation, the system exhibits a non-local character in the form of a pseudo-differential operator, which admits no equivalence to a beam-like system supported by any classical two-parameter elastic foundation. The paper is organized as follows: Sec.2 presents the equations of motion and the feature of an edge wave traveling solution. In Sec.3, the two sets of BCs are introduced and the corresponding dispersion relations obtained, together with the expressions for the phase and group velocity and the cut-off curve. An asymptotic analysis for a small deviation from the Winkler model is carried out in Sec.4 and an equivalent beam-like system is introduced in Sec.5. Finally, conclusions are drawn in Sec.6.

\section{Statement of the problem and traveling wave solution}

Let us consider a semi-infinite Kirchhoff plate with thickness $2 h$, bilaterally supported by a Pasternak two parameter elastic foundation. We investigate two cases corresponding to either a semi-infinite or an infinite foundation 


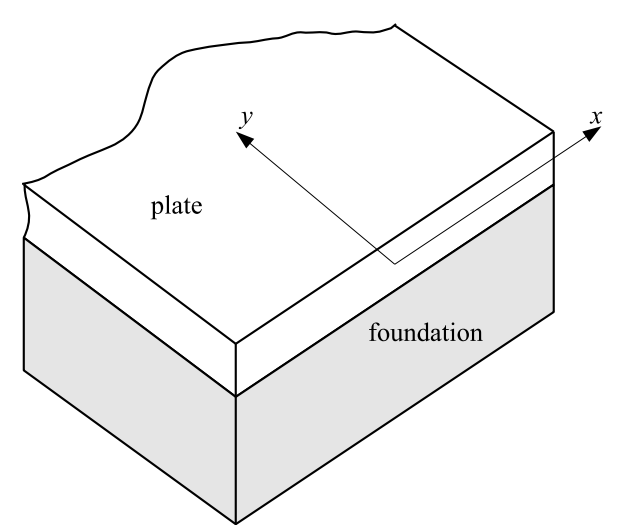

(a)

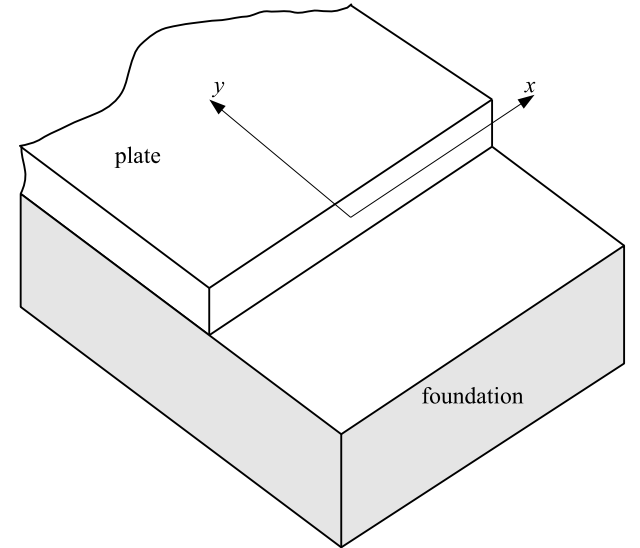

(b)

Figure 1: Semi-infinite Kirchhoff plate resting on a semi-infinite (a) or infinite (b) Pasternak elastic foundation

(Fig.1). The governing equation for the transverse displacement of the plate $w$ reads as

$$
D \triangle \triangle w-c \triangle w+\beta w+2 \rho h \partial_{t t} w=0,
$$

being $\triangle=\partial_{x x}+\partial_{y y}$ the Laplace operator in two dimensions, $D$ the plate bending stiffness and $\rho$ the mass density; the parameters $c$ and $\beta$ are usually named the Pasternak and the elastic modulus of the foundation. Eqs.(1) may be rewritten as

$$
\triangle \triangle w-2 \chi^{-2} \triangle w+\lambda^{-4}(w+\ddot{w})=0
$$

having let characteristic lengths

$$
\lambda=\sqrt[4]{\frac{D}{\beta}} \text { and } \quad \chi=\sqrt{\frac{2 D}{c}} .
$$


Here, dot denotes time differentiation with respect to the dimensionless time $\tau=t / \sqrt{2 \rho h / \beta}$. Letting the positive dimensionless ratio

$$
\eta=\frac{\lambda}{\chi}=\sqrt[4]{\frac{c^{2}}{4 \beta D}}
$$

together with dimensionless co-ordinates

$$
x_{1}=\lambda^{-1} x, \quad y_{1}=\lambda^{-1} y,
$$

Eq.(2) becomes

$$
\hat{\triangle} \hat{\triangle} w-2 \eta^{2} \hat{\triangle} w+w+\ddot{w}=0
$$

being $\hat{\triangle}$ the 2D Laplace operator in $x_{1}$ and $y_{1}$. Likewise, the governing equation for the soil transversal displacement $u$, in the absence of the plate (see Fig.1b), is given by

$$
-2 \eta^{2} \hat{\triangle} u+u=0
$$

Consider a traveling edge-wave solution

$$
w\left(x_{1}, y_{1}, \tau\right)=A \exp \left[i\left(K x_{1}-\Omega \tau\right)-\mu K y_{1}\right]
$$

Such edge-wave is propagating along the $x_{1}$-axis and it is exponentially decaying over the interior of the plate, i.e. for $y_{1}>0$, provided that $\Re(\mu)>0$. Here, $\mu$ is the attenuation index, $K=\lambda k$ where $k \in \mathbb{R}^{+}$is the wave number and $\Omega=\omega \sqrt{2 \rho h / \beta}$ where $\omega$ is the angular frequency. Plugging the traveling wave solution (5) into Eq.(2), we get the equation

$$
-\Omega^{2}+K^{4}\left(\mu^{2}-1\right)^{2}+1-2 \eta^{2} K^{2}\left(\mu^{2}-1\right)=0,
$$


which gives two possible solutions for $\mu$, namely

$$
\mu_{1,2}^{2}=\frac{\eta^{2} \mp \sqrt{\Omega^{2}+\eta^{4}-1}}{K^{2}}+1
$$

The decay condition $\Re\left(\mu_{1}\right)>0$ is satisfied provided that the parameters $K$ and $\Omega$ are such that the point $(K, \Omega)$ lies outside the region $\mathcal{R}$, being

$$
\mathcal{R}=\left\{(K, \Omega): \Omega^{2}-1+\eta^{4} \geq 0 \text { and } K^{2}+\eta^{2}-\sqrt{\Omega^{2}-1+\eta^{4}} \leq 0\right\} .
$$

Let us define

$$
z=\mu_{1} \mu_{2} .
$$

When $(K, \Omega) \in \mathcal{R}, \mu_{1}$ and $z$ are both either purely imaginary or zero. It is easy to prove that the converse holds true, which means that enforcing the decay condition $\Re\left(\mu_{1}\right)>0$ amounts to looking for $z$ to be real and positive.

Let us now consider an edge-wave solution for the soil in the form

$$
u\left(x_{1}, y_{1}, \tau\right)=B \exp \left[i\left(K x_{1}-\Omega \tau\right)+\gamma K y_{1}\right]
$$

which decays as $y_{1} \rightarrow-\infty$ provided that $\Re(\gamma)>0$. Plugging the solution (10) into Eq.(4), we have

$$
\gamma=\sqrt{1+\frac{1}{2 \eta^{2} K^{2}}}
$$

\section{Dispersion relations}

We now discuss separately the two set of BCs relating to a semi-infinite and infinite foundation (Fig.1). 


\subsection{Semi-infinite foundation}

The BCs on the free plate edge $y=0$ need take into account the presence of the a line loading arising from the foundation (Nobili, 2012b; Selvadurai, 1979), namely

$$
m_{y}=0, \quad v_{y}+c \partial_{y} w=0,
$$

being

$$
m_{y}=-D\left(\partial_{y y}+\nu \partial_{x x}\right) w, \quad v_{y}=-D\left[\partial_{y y y}+(2-\nu) \partial_{x x y}\right] w
$$

respectively the bending moment and the Kirchhoff equivalent shearing force. Here, $\nu$ is the Poisson ratio. In dimensionless co-ordinates, the second BC yields

$$
-\hat{v}_{y}+2 \eta^{2} \partial_{y_{1}} w=0
$$

holding at $y_{1}=0$. Here, we let $\hat{v}_{y}=\left[\partial_{y_{1} y_{1} y_{1}}+(2-\nu) \partial_{x_{1} x_{1} y_{1}}\right] w$.

We satisfy the BCs using a linear combination of two traveling waves, namely

$$
w=A_{1} \exp \left[i\left(K x_{1}-\Omega \tau\right)-\mu_{1} K y_{1}\right]+A_{2} \exp \left[i\left(K x_{1}-\Omega \tau\right)-\mu_{2} K y_{1}\right]
$$

whence we require

$$
\begin{aligned}
A_{1} K^{2}\left(\mu_{1}^{2}-\nu\right)+A_{2} K^{2}\left(\mu_{2}^{2}-\nu\right) & =0 \\
K^{2}\left[A_{1} \mu_{1}\left(\mu_{1}^{2}+\nu-2\right)+A_{2} \mu_{2}\left(\mu_{2}^{2}+\nu-2\right)\right] & =2 \eta^{2}\left(A_{1} \mu_{1}+A_{2} \mu_{2}\right) .
\end{aligned}
$$

This homogeneous system of linear equations in $A_{1}, A_{2}$ has solutions other than the trivial one provided that

$$
z^{2}+2\left(1-\nu+\frac{\eta^{2}}{K^{2}}\right) z-\nu^{2}=0
$$


Clearly, Eq.(14) possesses two roots $z_{1}<0<z_{2}$, the second of which is always admissible. Substituting for $z$ via Eqs. $(7,9)$, we find the dispersion relation in terms of $K$ and $\Omega$

$$
f(K, \Omega)=0,
$$

where

$f(K, \Omega)=1-\Omega^{2}+K^{4}\left(1-\nu^{2}\right)+2 K^{2} \eta^{2}+2\left[(1-\nu) K^{2}+\eta^{2}\right] \sqrt{1+K^{4}+2 K^{2} \eta^{2}-\Omega^{2}}$,

which, upon solving for the square root and squaring, can be presented in explicit form

$$
\Omega^{2}=1-K^{4}(1-\nu)(1-3 \nu)+2 K^{2}\left[(1-\nu) R-\eta^{2}(1-2 \nu)\right]+2 \eta^{2}\left(R-\eta^{2}\right),
$$

where

$$
\nu_{0}=\sqrt{2 \nu^{2}-2 \nu+1}, \quad R=\sqrt{\nu_{0}^{2} K^{4}+2(1-\nu) \eta^{2} K^{2}+\eta^{4}} .
$$

When $\eta=0$, Eq.(16) reduces to (19) of Kaplunov et al. (2014) which corresponds to the Winkler foundation, i.e.

$$
\gamma_{e}^{4} K^{4}=\Omega^{2}-1
$$

where

$$
\gamma_{e}=\sqrt[4]{(1-\nu)\left(3 \nu-1+2 \nu_{0}\right)}
$$

is a known edge-wave constant, cf. Lawrie and Kaplunov (2012). Fig.2 plots $\Omega$ as a function of $K$ according to Eq.(16) for $\eta=0,0.5,1$ at $\nu=0.4$. It is 
easy to see that all curves converge to the cut-off frequency $\Omega_{0}=1$ for the Winkler case, for at the long wave regime

$$
\Omega^{2}=1+2 \eta^{2} K^{2}+O\left(K^{4}\right)
$$

The phase velocity $V^{p h}=\Omega / K$ is easily obtained from (16), namely $V^{p h}=\frac{\sqrt{1-K^{4}(1-\nu)(1-3 \nu)+2 K^{2}\left[(1-\nu) R-\eta^{2}(1-2 \nu)\right]+2 \eta^{2}\left(R-\eta^{2}\right)}}{K}$.

The group velocity is given by

$$
V^{g}=\frac{d \Omega}{d K}=2\left\{\eta^{2}(2 \nu-1)-2(\nu-1) s+K^{2}\left[\frac{\nu^{2}}{s} \eta^{2}-3 \nu^{2}+4 \nu-1\right]\right\}\left(V^{p h}\right)^{-1}
$$

Fig.3 plots the phase and group velocity curves for $\eta=0,0.5,1$ at $\nu=0.4$.

Phase velocity $V^{p h}$ exhibits a minimum point where it intersects the group velocity curve. Indeed, $V^{p h} \sim K^{-1}$ as $K \rightarrow 0^{+}$and $V^{p h} \sim \gamma_{e}^{2} K$ as $K \rightarrow+\infty$ (cfr. Eq.(26)).

\subsection{Infinite foundation}

The BCs on the plate free edge $y=0$ are given in Nobili (2012b)

$$
w=u, \quad m_{y}=0, \quad v_{y}+c\left(\partial_{y} w-\partial_{y} u\right)=0,
$$

where $u$ is the displacement field for the infinite foundation. The BC on the shearing force may be rewritten as

$$
-\hat{v}_{y}+2 \eta^{2}\left(\partial_{y_{1}} w-\partial_{y_{1}} u\right)=0
$$




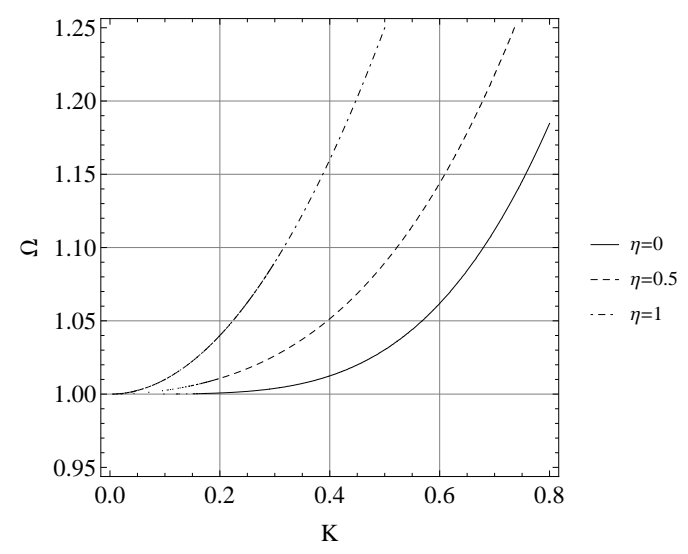

Figure 2: Dispersion curves for edge-waves of a plate resting on a semi-infinite Pasternak foundation $(\nu=0.4)$

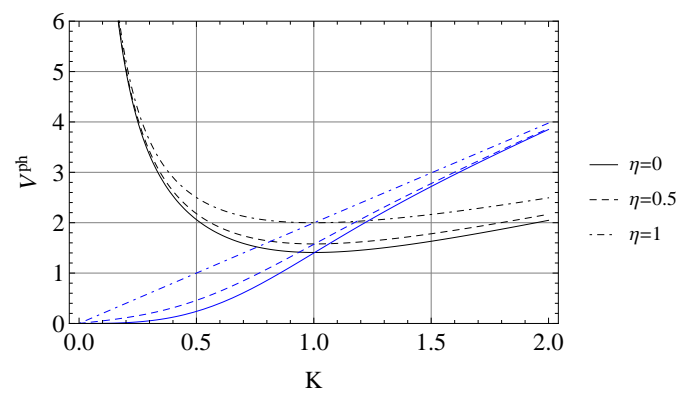

Figure 3: Phase and group velocity curves of a plate resting on a semi-infinite foundation $(\nu=0.4)$ 
We satisfy the BCs using a linear combination of three waves, namely Eq.(13) for the plate and Eq.(10) for the soil. Enforcing the conditions (20) yields a homogeneous system of linear equations in $A_{1}, A_{2}, B$ whose non-trivial solutions exist provided that

$$
K^{2}\left[z^{2}+2(1-\nu) z-\nu^{2}\right]+2 \eta^{2}\left[z+\gamma \sqrt{2\left(1+\frac{\eta^{2}}{K^{2}}\right)+2 z}\right]=0 .
$$

Here we have used Eq.( 7$)$ to get $\mu_{1}^{2}+\mu_{2}^{2}=2\left(1+\eta^{2} / K^{2}\right)$. Plugging in Eq.(11) for $\gamma$, we arrive at the dispersion relation for $z$ and $K$

$$
\begin{aligned}
K^{2}\left[z^{2}+2\left(1-\nu+\frac{\eta^{2}}{K^{2}}\right)\right. & \left.z-\nu^{2}\right]+ \\
& +2 \frac{\eta}{K^{2}} \sqrt{\left(2 \eta^{2} K^{2}+1\right)\left(\eta^{2}+K^{2}(z+1)\right)}=0 .
\end{aligned}
$$

It is observed that the term in square brackets corresponds to the dispersion relation given in Eq.(14) so that the infinite nature of the foundation contributes only with the last term. This equation admits one positive root for $z$ given that

$$
p(m)=4 m^{2}(1+m)\left(2+\eta^{-4} m\right)-\nu^{4}<0, \quad m=\frac{\eta^{2}}{K^{2}}>0 .
$$

The inequality (22) follows from inspection of Eq.(21) at $z=0$. In the limiting case $\eta=0$, this inequality is always satisfied for any $K>0$. Let $m_{0}$ be the single positive root of the equation

$$
p\left(m_{0}\right)=0,
$$

then

$$
K>K_{0}=\frac{\eta}{\sqrt{m_{0}}} \geq 0
$$


and

$$
\Omega>\Omega_{0}=\sqrt{1+\eta^{4}\left[\left(1+\frac{1}{m_{0}}\right)^{2}-1\right]} \geq 1,
$$

equality holding only in the case $\eta=0$. Substituting for $z$ through Eqs. $(7,9)$, we get the dispersion relation in terms of $K$ and $\Omega$

$$
f(K, \Omega)+2 \eta \sqrt{\left(2 \eta^{2} k^{2}+1\right)\left(\eta^{2}+K^{2}+\sqrt{K^{4}+2 \eta^{2} K^{2}-\Omega^{2}+1}\right)}=0,
$$

where $f(K, \Omega)$ is defined in (15). Fig.4 plots the dispersion curve (24) together with the cut-off points $\left(\Omega_{0}, K_{0}\right)$, for $\eta=0,0.05,0.1$ and $\nu=0.4$. Note that $\Omega_{0} \geq 1$ and the equality holds in the case $\eta=0$, that is for the Winkler foundation, in which case $K_{0}=0$. Already at fairly small values of $\eta, m_{0} \ll 1$. Eq.(21) reduces to (13) of Kaplunov et al. (2014) whenever $\eta=0$. Conversely, the corresponding dispersion relation (14), valid for the semi-infinite foundation, cannot be retrieved as a limiting case of Eq.(21). Fig.5 plots the phase velocity $V^{p h}$ again for $\eta=0,0.05,0.1$ and $\nu=0.4$.

\section{Asymptotic expansions}

In the case $\eta \ll 1$, i.e. the near-Winkler regime, the dispersion relation may be given an asymptotic expansion in $\eta$.

\subsection{Semi-infinite foundation}

Eq.(16) may be expanded up to second order terms in $\eta$ as

$$
\Omega^{2}=1+K^{4} \gamma_{e}^{4}+2 \psi_{1} K^{2} \eta^{2}+O\left(\eta^{4}\right)
$$




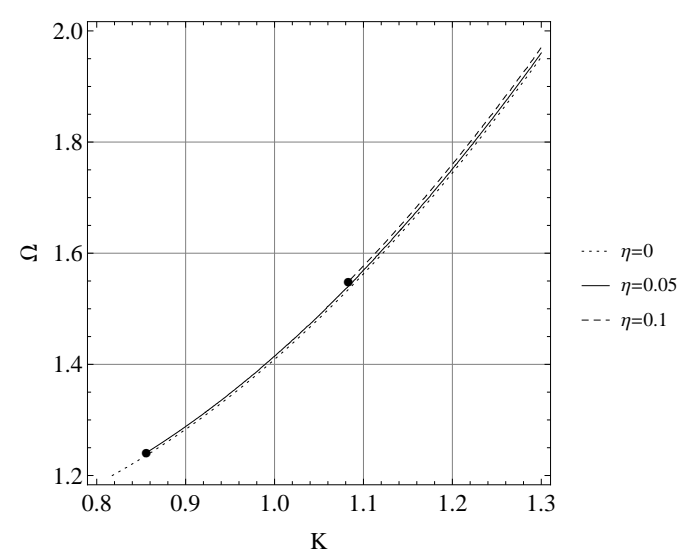

Figure 4: Dispersion curves and cut-off points $\left(K_{0}, \Omega_{0}\right)$ for edge-waves of a plate resting on an infinite Pasternak foundation $(\nu=0.4)$

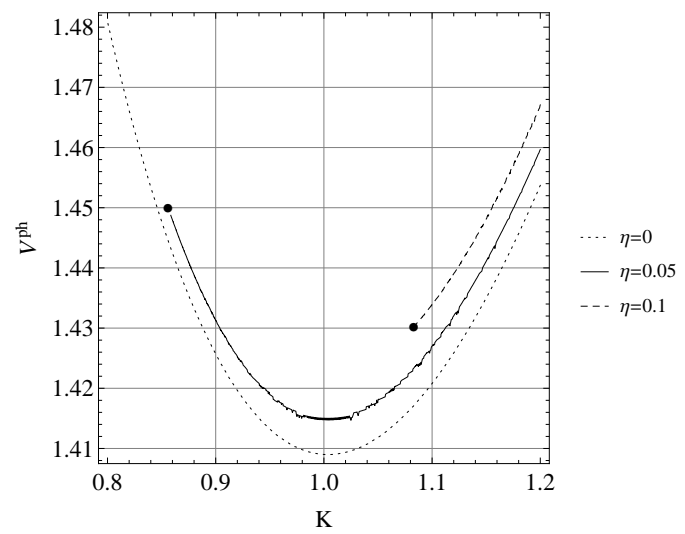

Figure 5: Phase velocity and cut-off points $\left(K_{0}, V_{0}^{p h}\right)$ for edge-waves of a plate resting on an infinite Pasternak foundation $(\nu=0.4)$ 
where $\psi_{1}=-1+2 \nu+\frac{2-4 \nu+3 \nu^{2}}{\nu_{0}}$. Consequently, the phase velocity is simply

$$
V^{p h}=\frac{\sqrt{1+\gamma_{e}^{4} K^{4}}}{K}+\frac{\psi_{1}}{\sqrt{1+\gamma_{e}^{4} K^{4}}} K \eta^{2}+O\left(\eta^{4}\right) .
$$

It can be easily shown that the position of the phase velocity minimum occurs at

$$
K_{*}=\gamma_{e}^{-1}+O\left(\eta^{2}\right)
$$

which supports a weak dependance of this value on $\eta$, as shown in Fig.3. The corresponding minimal phase velocity is given by

$$
V_{*}=\sqrt{2} \gamma_{e}+O\left(\eta^{2}\right) .
$$

It follows that within the truncation error, $K_{*}$ and $V_{*}$ coincide with their counterparts given in Kaplunov et al. (2014) for the Winkler foundation.

\subsection{Infinite foundation}

Eq.(24) cannot be easily solved explicitly for $\Omega^{2}$. However, up to first order terms in $\eta$, it can be replaced by

$K^{4}\left(\nu^{2}-1\right)+2 K^{2}(\nu-1) \sqrt{K^{4}-\Omega^{2}+1}+\Omega^{2}-1-2 \eta \sqrt{\sqrt{K^{4}-\Omega^{2}+1}+K^{2}}+O\left(\eta^{2}\right)=0$

and when dealing with a first order approximation in $\eta$, the $\eta$ term can be taken at the leading order approximation (17), namely

$K^{4}\left(\nu^{2}-1\right)+2 K^{2}(\nu-1) \sqrt{K^{4}-\Omega^{2}+1}+\Omega^{2}-1-2 \eta K \sqrt{1+\sqrt{1-\gamma_{e}^{4}}}+O\left(\eta^{2}\right)=0$.

Now eliminating the square root and solving for $\Omega^{2}$ as for the semi-infinite setup dealt with in Sec.3.1, we can rewrite the previous dispersion relation 
in an explicit form

$$
\Omega^{2}=1+K^{4} \gamma_{e}^{4}+2 \psi_{2} K \eta+O\left(\eta^{2}\right)
$$

where $\psi_{2}=\left(1-\frac{1-\nu}{\nu_{0}}\right) \sqrt{1+\sqrt{1-\gamma_{e}^{4}}}$. The corresponding phase velocity is given by

$$
V^{p h}=\frac{\sqrt{1+\gamma_{e}^{4} K^{4}}}{K}+\frac{\psi_{2}}{\sqrt{1+\gamma_{e}^{4} K^{4}}} \eta+O\left(\eta^{2}\right)
$$

and its minimum is

$$
\min _{K} V^{p h}=V_{*}=\sqrt{2} \gamma_{e}+\frac{\psi_{2}}{\sqrt{2}} \eta+O\left(\eta^{2}\right)
$$

which is attained at

$$
K_{*}=\frac{1}{\gamma_{e}}+\frac{\psi_{2}}{4 \gamma_{e}^{2}} \eta+O\left(\eta^{2}\right) \quad \text { and } \quad \Omega_{*}=\sqrt{2}+\frac{3 \psi_{2}}{2 \sqrt{2} \gamma_{e}} \eta+O\left(\eta^{2}\right) .
$$

Figs. 6 plots the cut-off curves in the $\eta, K$ plane for three different values of $\nu$ and the corresponding asymptotic expansions for $\eta$ small, namely

$$
m_{0} \sim \sqrt[3]{\frac{\nu^{4} \eta^{4}}{4}}, \quad \eta \ll 1,
$$

leading to

$$
K_{0} \sim \sqrt[3]{\frac{2 \eta}{\nu^{2}}}, \quad \Omega_{0} \sim 1
$$

From Eqs.(29,31), a limiting value for $\eta$ may be defined, namely

$$
\eta_{0}=\frac{\nu^{2}}{2 \gamma_{e}^{3}}
$$

for which $K_{0}=\gamma_{e}^{-1}$. For $\eta>\eta_{0}$, the minimum of the phase velocity is attained outside the admissibility region for $K$. It is observed that, strictly speaking, from the standpoint of asymptotic theory, the reasoning leading 


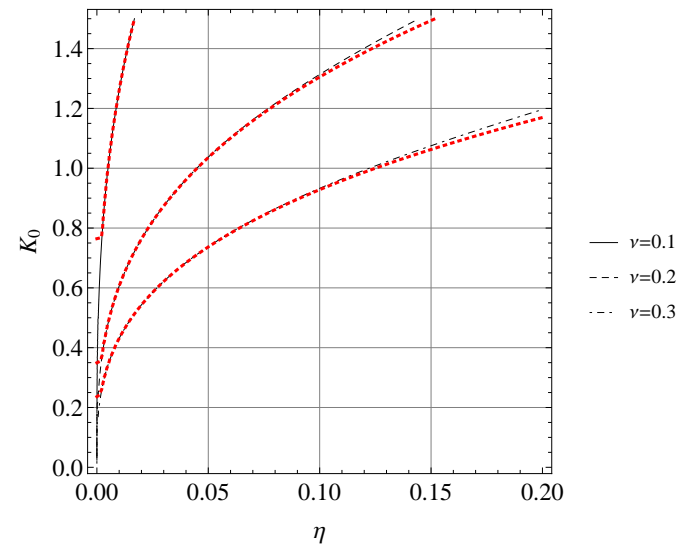

Figure 6: Cut-off wavenumber $K_{0}$ vs. $\eta$ and its approximation (dotted curves) as given by Eq.(31)

to $\eta_{0}$ is formally incorrect, for the asymptotic expansions are obtained in the assumption that $\eta$ is small, in particular $\eta \ll \eta_{0}$. Nonetheless, $\eta_{0}$ is a valid numerical approximation for the validity region for $K$. Fig.7 plots the wavenumber cut-off $K_{0}$ as well as the value $K_{*}$ corresponding to the phase velocity minimum vs. the parameter $\eta$ at $\nu=0.4$. In this Figure, the asymptotic formula (31) is compared to the "exact" value $K_{0}=\frac{\eta}{\sqrt{m_{0}}}$ obtained through a numerical solution of Eq.(23). Similarly, the leading order approximation $K_{*}=\gamma_{e}^{-1}$ (cf.(29)) is plotted against the curve computed via the dispersion relation (24). The intersection between the asymptotic curves for $K_{0}$ and $K_{*}$ yields $\eta_{0}$. 


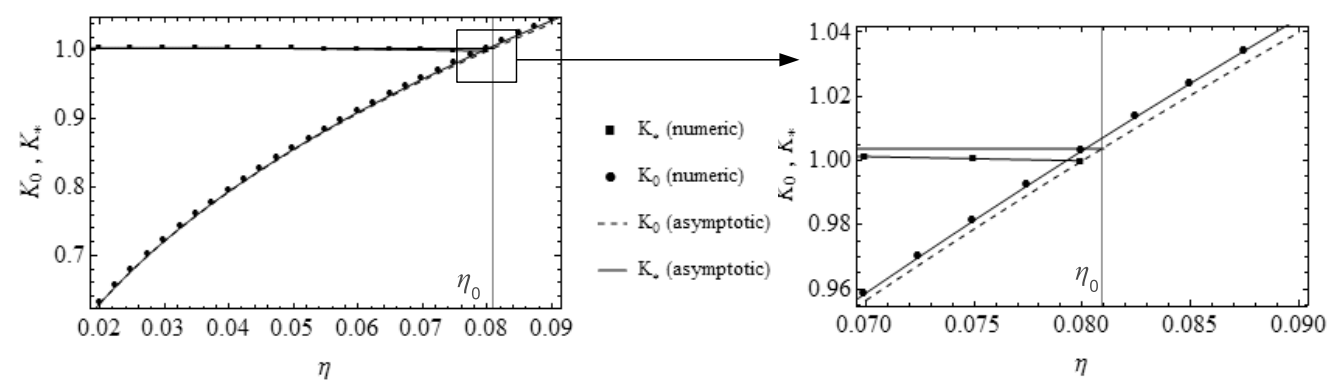

Figure 7: Asymptotics vs. numerics for the cut-offs and phase velocity minima of a plate resting on an infinite Pasternak foundation

\section{Beam-like models for edge waves}

Following Kaplunov et al. (2014), the asymptotic dispersion relations (25, 27) may be interpreted in terms of a beam resting on an elastic soil. For a semi-infinite foundation, the soil behaves like a Pasternak foundation and the equation of motion of such a system becomes

$$
D_{b} \partial_{x x x x} w+2 \rho h \partial_{t t} w-c_{b} \partial_{x x} w+\beta_{b} w=0,
$$

where $D_{b}$ is the effective beam flexural rigidity, $c_{b}$ and $\beta_{b}$ the effective Pasternak and Winkler moduli, respectively. Dynamic equivalence demands

$$
D_{b}=D \gamma_{e}^{4}, \quad c_{b}=c \psi_{1}, \quad \beta_{b}=\beta
$$

This equation supports a critical velocity of a moving load which coincides with the minimal phase velocity $V_{*}$, e.g. Timoshenko (1926). It is worth mentioning that a critical velocity is also characteristic of edge moving perturbances acting on a plate supported by a foundation with non-small Pasternak coefficient for which reduction to a beam-like system is impossible. It can be 
verified that the last identity in Eqs.(33) is refined at the next asymptotic order for the expansion (25). Also, the high-order expansions of the latter bring in inverse powers of the wavenumber $K$, which hints that a non-local foundation model is dealt with. Such non-local feature is even more pronounced in the infinite foundation model. In this case, the two term expansion (27) corresponds to the following equation of motion:

$$
D_{b} \partial_{x x x x} w+2 \rho h \partial_{t t} w+p_{b} \sqrt{-\partial_{x x}} w+\beta_{b} w=0,
$$

where $\sqrt{-\partial_{x x}}$ is a pseudo-differential operator associated with the non-local feature of the foundation (Dai et al., 2010). Here,

$$
p_{b}=\sqrt{2 c \beta} \text {. }
$$

Thus, the two-parameter elastic foundation is no longer of the Pasternak type. Furthermore, the range of validity of the beam like system underlying Eq.(34) is restricted to the wavenumbers above the nonzero cut-off $K_{0}$, as discussed in the previous Section.

\section{Conclusions}

The dispersion relations for edge bending waves on a semi-infinite Kirchhoff plate supported by a two parameter Pasternak foundation are derived. Two layouts are considered for the foundation: either it is infinite or it is semiinfinite and its edge coincides with the edge of the plate. The phase and group velocity are expressed in terms of a single dimensionless parameter expressing 
the relative stiffness of the foundation. The main focus is set on the cut-off frequencies and on the minima of the phase velocity, the latter corresponding to the critical velocities of a moving load. Asymptotic expansions for a nearly local (Winkler) foundation are presented. As a physical consequence, similarity of edge plate waves and bending waves on elastically supported beams is established.

Treatment of the infinite Pasternak foundation reveals several unexpected features, including a sophisticated dependence of the cut-off frequency and of the minimum of the phase velocity on the relative soil stiffness. It is also remarkable that the cut-off frequency is observed at a nonzero wavenumber, which is a rather rare occurrence in elastodynamics. As an example, we mention the first symmetric Lamb harmonics in an elastic layer, e.g. see Achenbach (1984). Furthermore, the edge wave on a plate resting on a infinite foundation may be only interpreted in terms of a beam laying on a nonclassical foundation modeled by a pseudo-differential operator.

The obtained results may be extended to anisotropic and pre-stressed structures and also adapted to refined plate models. In addition, analysis of a plate supported by more general types of infinite foundation, possibly including an elastic half-space, seems to be of obvious theoretical and practical interest. A similar problem for an acoustic half space was addressed in Abrahams and Norris (2000). 


\section{Acknowledgments}

This work was supported by "Fondazione Cassa di Risparmio di Modena" within the framework of the Progetti di Ricerca finalizzata all'innovazione 2014, Sime n.2013.0662. JK gratefully acknowledges support from Modena University through the Long Visiting Professor programme 2014. AN gratefully acknowledges support from the National Group of Mathematical Physics (GNFM-INdAM) through the "Progetto Giovani Ricercatori 2015" scheme, prot. U2015/000125.

\section{References}

Abrahams, I. and Norris, A. (2000), On the existence of flexural edge waves on submerged elastic plates, Proceedings of the Royal Society of London. Series A: Mathematical, Physical and Engineering Sciences 456(1999), 1559-1582.

Achenbach, J. (1984), Wave propagation in elastic solids, Elsevier.

Bell, A. (2006), Sensors, motors, and tuning in the cochlea: interacting cells could form a surface acoustic wave resonator, Bioinspiration $\&$ biomimetics $1(3), 96$.

Biryukov, S., Gulyaev, Y., Krylov, V. and Plessky, V. (1995), Surface acoustic waves in inhomogeneous media, Vol. 20, Springer.

Dai, H.-H., Kaplunov, J. and Prikazchikov, D. (2010), A long-wave model for 
the surface elastic wave in a coated half-space, Proceedings of the Royal Society A: Mathematical, Physical and Engineering Science 466(2122), 30973116.

Ishlinskii, A. (1954), On a limiting process in the theory of the stability of elastic rectangular plates, Dokl. Akad. Nauk SSSR 95(3), 477-479.

Kaplunov, J. and Prikazchikov, D. (2013), Explicit models for surface, interfacial and edge waves, in 'Dynamic Localization Phenomena in Elasticity, Acoustics and Electromagnetism', Springer, pp. 73-114.

Kaplunov, J., Prikazchikov, D., Rogerson, G. and Lashab, M. (2014), The edge wave on an elastically supported Kirchhoff plate, The Journal of the Acoustical Society of America 136(4), 1487-1490.

Konenkov, Y. (1960), A Rayleigh-type flexural wave, Sov. Phys. Acoust $6,122-123$

Lawrie, J. and Kaplunov, J. (2012), Edge waves and resonance on elastic structures: An overview, Mathematics and Mechanics of Solids 17(1), 416.

Nobili, A. (2012a), Superposition principle for the tensionless contact of a beam resting on a Winkler or a Pasternak foundation, Journal of Engineering Mechanics 139(10), 1470-1478.

Nobili, A. (2012b), Variational approach to beams resting on two- 
parameter tensionless elastic foundations, Journal of Applied Mechanics $79(2), 021010$.

Norris, A., Krylov, V. and Abrahams, I. (2000), Flexural edge waves and comments on "A new bending wave solution for the classical plate equation" [j. acoust. soc. am. 104, 2220-2222 (1998)], The Journal of the Acoustical Society of America 107(3), 1781-1784.

Sallenger Jr, A. (1979), Beach-cusp formation, Marine Geology 29(1), 23-37.

Selvadurai, A. (1979), Elastic analysis of soil-foundation interaction, Vol. 17 of Developments in geotechnical engineering, Elsevier scientific publishing company.

Timoshenko, S. (1926), Method of analysis of statical and dynamical stresses in rail, in 'Proc. Second Int. Congress of Appl. Mech., Zurich', pp. 12-17.

White, R. (1970), Surface elastic waves, Proceedings of the IEEE 58(8), 12381276.

White, R. and Voltmer, F. (1965), Direct piezoelectric coupling to surface elastic waves, Applied Physics Letters 7(12), 314-316.

Wieghardt, K. (1922), Über den Balken auf nachgibiger Unterlage, Zeitschrift für angewandte Mathematik und Mechanik (ZAMM) 2(3), 165-184. 